\title{
Ehlers-Danlos syndrome VIII with novel C1R variant accompanying white matter changes
}

\author{
Go Hun Seo', Yoon-Myung Kim², Byeongzu Ghang ${ }^{3}$, Gu-Hwan Kim, and Beom Hee Lee, ${ }^{1,4, *}$ \\ ${ }^{1}$ Department of Pediatrics, Asan Medical Center, University of Ulsan College of Medicine, Seoul, Korea \\ ${ }^{2}$ Department of Pediatrics, Jeju National University School of Medicine, Jeju, Korea \\ ${ }^{3}$ Department of Rheumatology, Asan Medical Center, University of Ulsan College of Medicine, Seoul, Korea \\ ${ }^{4}$ Medical Genetics Center, Asan Medical Center, University of Ulsan College of Medicine, Seoul, Korea
}

\begin{abstract}
Ehlers-Danlos syndrome (EDS) VIII is an autosomal dominant inherited connective tissue disorder characterized by intractable periodontal inflammation, absence of gingiva, pretibial plaques, skin hyperextensibility, joint hypermobility, and tissue fragility with onset in the childhood or adolescence. In a recent report, heterozygous variants of the C1R or C1S related to the classical complement pathway were identified in families with history of EDS VIII. The current report describes a Korean 34-year-old female carrying a novel missense variant of C1R c.925T>G (p.Cys309Gly) and exhibiting early severe periodontitis, skin fragility, and joint hypermobility. The patient also had frontal, parietal, and temporal white matter brain lesions without definite vascular abnormalities on brain magnetic resonance imaging, which have not been surveyed meticulously in EDS VIII. Considering the genetic alteration of classic complement pathways in this condition, it is necessary to carefully observe multisystemic inflammation processes such as changes in brain white matter.
\end{abstract}

Key words: Ehlers-Danlos syndrome type VIII, Complement C1r, White matter.

\section{Introduction}

Ehlers-Danlos syndromes (EDS) are inherited connective tissue disorders characterized by phenotypic heterogeneity and genetic diversity, showing the common features of skin hyperextensibility, joint hypermobility, and tissue fragility [1-3]. EDS VIII (OMIM 130080) was first reported in 1972 in a patient diagnosed with early severe periodontitis, skin fragility, and abnormal scarring, which were subsequently recognized as symptoms of a distinct EDS subtype inherited an autosomal dominant manner in 1977 [4]. The cardinal features of EDS VIII are severe and intractable periodontal inflammation, pretibial plaques with onset in childhood or adolescence, and a family history of first-degree relatives $[1,3]$.

The $7 \mathrm{cM}(5.8 \mathrm{MB})$ interval on chromosome $12 q 13$ locus has evidence for linkage with logarithm of the odds score 5.17 for EDS VIII [5]. Genome-wide linkage scan performed in a 4-generation family with history of EDS VIII detects linked region on chromosome 9 [6]. However, any candidate gene in these regions has not been identified $[5,6]$. In a recent report, heterozygous variants of the C1R (OMIM 613785) or C1S (OMIM 120580) located on 12p13 related to the classical complement pathway were identified in 17 families with history of EDS VIII (98 individuals) using whole exome sequencing [7].

Received: 19 September 2018, Revised: 12 October 2018, Accepted: 15 October 2018, Published: 30 June 2019

${ }^{*}$ Corresponding author: Beom Hee Lee, M.D., Ph.D. (iD https://orcid.org/0000-0001-9709-2631

Department of Pediatrics, Asan Medical Center, University of Ulsan College of Medicine, 88 Olympic-ro 43-gil, Songpa-gu, Seoul 05505, Korea.

Tel: +82-2-3010-5950, Fax: +82-2-473-3725, E-mail: bhlee@amc.seoul.kr

Conflict of interest: The authors declare that they do not have any conflicts of interest.

(ac) This is an open-access article distributed under the terms of the Creative Commons Attribution Non-Commercial License (http://creativecommons.org/licenses/by-nc/4.0/) which permits unrestricted non-commercial use, distribution, and reproduction in any medium, provided the original work is properly cited.

(c) Copyright 2019 by the Korean Society of Medical Genetics and Genomics 
The current report describes a Korean female carrying a novel missense variant of $C 1 R$ exhibiting early severe periodontitis, skin fragility, and joint hypermobility. Of note, she had multifocal white matter brain lesions, which have not been surveyed meticulously in EDS VIII [8].

\section{Case}

A 34-year-old female came to the Asan medical center rheumatologic outpatient clinic due to facial rash and abnormal findings on brain magnetic resonance imaging (MRI), which was done for the evaluation of general fatigue and intermittent headaches. Neurological examinations of the motor and sensory nervous system and cranial nerves had normal results. She had taken thyroid hormone for 10 years due to hypothyroidism and received extracorporeal shock wave lithotripsy 3 times due to left renal stone. She smoked one pack of cigarettes a day for five years. Brain MRI showed bilateral patchy T2-weight high-signal intensity lesions in frontal, parietal, and temporal white matter without definite vascular abnormalities (Fig. 1A). Brain magnetic resonance angiography showed no definite vascular abnormalities (Fig. 1B). Laboratory studies to rule out vasculitis associated

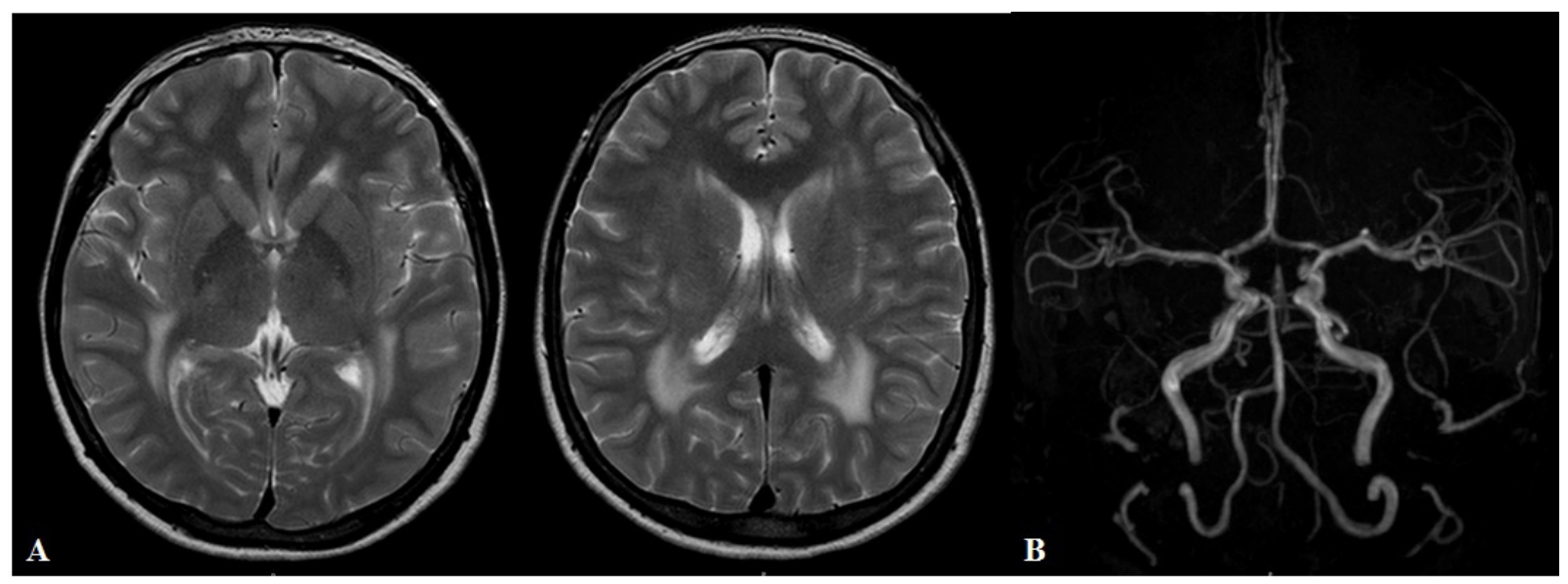

Fig. 1. (A) Brain magnetic resonance imaging showed bilateral patchy T2-weight fluid-attenuated inversion recovery high-signal intensity lesions in frontal, parietal, and temporal white matter. (B) There are no definite vascular abnormalities on the brain magnetic resonance angiography.
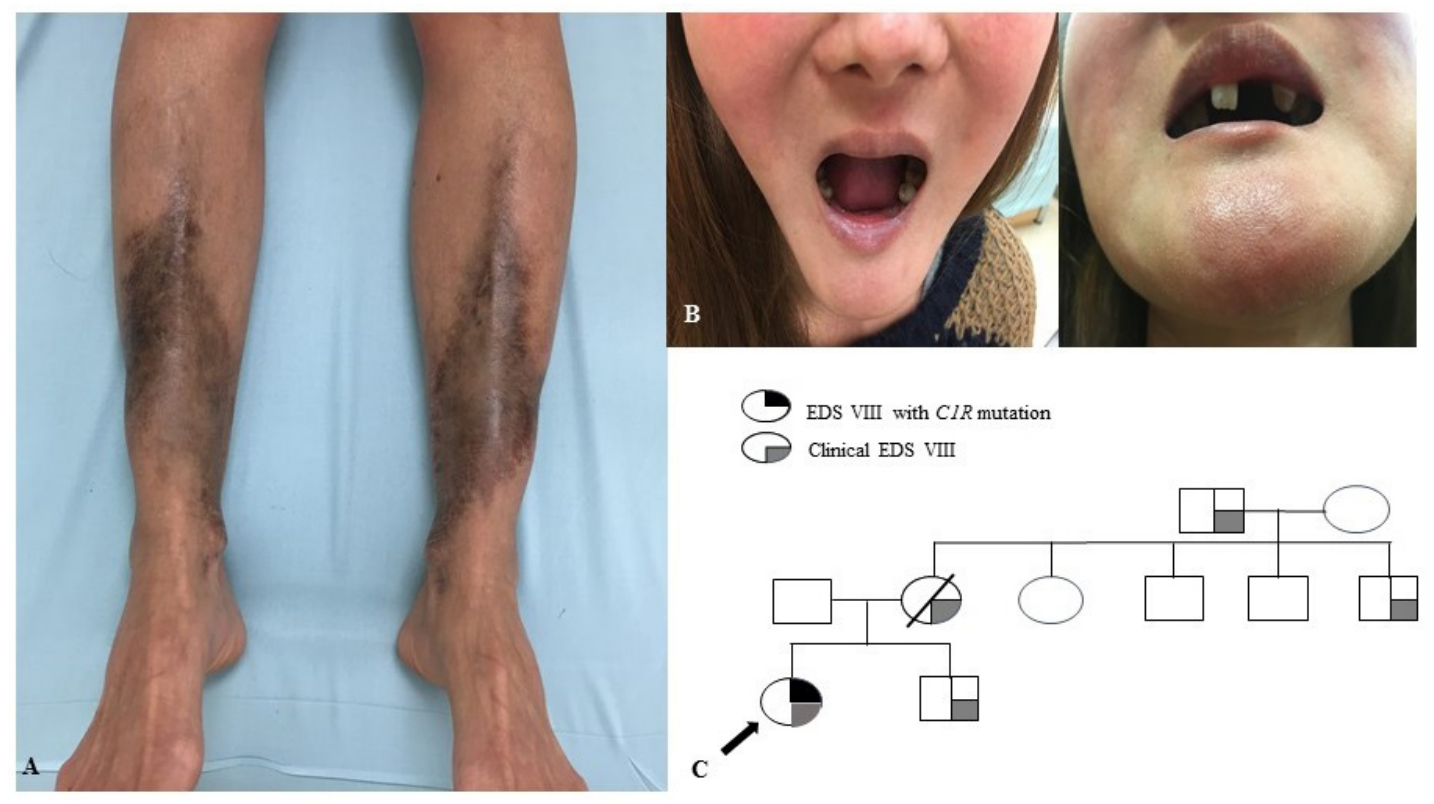

Fig. 2. (A) Brownish hyperpigmentation of both shins. (B) The patient's remaining teeth. (C) Pedigree for the patient (arrow: proband). EDS, Ehlers-Danlos syndrome. 
with autoimmune diseases showed antinuclear antibody, antineutrophil cytoplasmic antibody, cryoglobulin, rheumatoid factor, anti-Sjögren's syndrome A, anti-Sjögren's syndrome B, antithyroid peroxidase antibody, thyroid function, complete blood count, and coagulation profile to be normal.

The patient was referred to the medical genetics clinic due to suspicion of connective tissue disorders because her legs were susceptible to bruising and recovery had been slow since early childhood. Physical examination revealed marked skin fragility with easy bruising, brownish hyperpigmentation of both shins (Fig. 2A), facial rash, and skin hyperextensibility. She showed hypermobility of both the elbow and knee joints, and the Beighton score was 5 . She was diagnosed with periodontitis at the age of 20 . Since then, she has been suffering from recurrent periodontal infections and has begun to lose her teeth. To date, she has lost most of her teeth except for six molars (Fig. 2B), which have been replaced by artificial teeth. She had no dysmorphism including triangular facial structure, prominent eyes, and a long nose. Her voice was hoarse. The patient recalled that her mother had lost all of her teeth by her early 20s and also had pretibial bruising. She ultimately died of gastric adenocarcinoma at the age of 40 years. Her youngest maternal uncle also suffered from periodontal problems, and her younger brother had lost all of his teeth by his early 30s and showed pretibial bruising (Fig. 2C).

Informed consent was obtained, and blood sample was collected from the patient. Genomic DNA was extracted from peripheral blood leukocytes using a Puregene DNA isolation kit (Gentra, Minneapolis, MN, USA). All coding 11 exons and exonintron boundaries of $C 1 R$ were directly sequenced using an $A B I$ 3130 genetic analyzer (Applied Biosystems, Foster City, CA, USA) and a BigDye Terminator cycle sequencing kit (Applied Biosystems) in peripheral leukocytes. A previously unknown germ line variant, c.925T>G (p.Cys309Gly), was identified in the $C 1 R$ (Fig. 3). The variant p.Cys309Gly, which has not been reported in the general population (http://gnomad.broadinstitute.org/about), was predicted to be pathogenic by in silico prediction programs such as a PolyPhen-2 (http://genetics.bwh.harvard.edu/pph2/) score of 1 and Sorting Intolerant from Tolerant (http://sift.jcvi. $\mathrm{org} / \mathrm{l}$ score of 0 . This variant is classified as likely to be pathogenic according to the American College of Medical Genetics and Genomics guideline [9].

After the confirmation of the diagnosis of EDS VIII, echocardiography was done in order to check for vascular and cardiac problems and gave normal results. Smoking cessation and regular dental examination were recommended for the care of periodontitis.

\section{Discussion}

More than $90 \%$ of patients with EDS VIII exhibit early severe periodontitis, gingival recession, absence of attached gingiva, and easy bruising [7]. Pretibial hyperpigmentation, skin fragility, and elastic skin are also common, and abnormal scarring, joint hypermobility, recurrent infection, and marfanoid facial features may be observed $[3,7,10]$. Rarely, autoimmune disorder, organ rupture, osteoarthritis, hoarseness, and joint dislocation were also reported in EDS VIII $[7,11]$. EDS VIII can be suspected based on these distinctive clinical features $[3-6,10,11]$. The patient described in the current report exhibited these distinct features via dominant inheritance from her mother as well as atypical feature such as hoarse voice.

The pathogenic mechanism of EDS VIII has thus far been elusive. However, considering that the genetic defect of EDS VIII resides in the first component of the classical complement path-

\section{Partial sequence of $C 1 R$ gene}

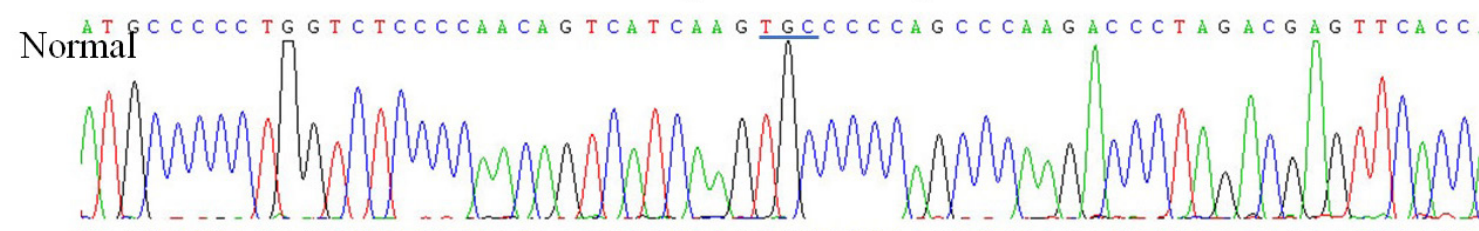

Patient

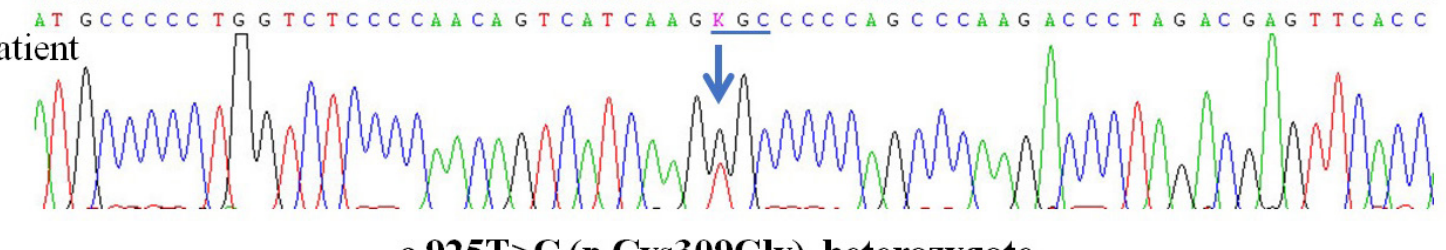

\section{c.925T $>$ G (p.Cys309Gly), heterozygote}

$\mathrm{K}=\mathrm{T}$ and $\mathrm{G}$

Fig. 3. The novel missense variant, c.925T>G (p.Cys309Gly), in the C1R of the patient. 
way, C1 complex [7], its pathologic features might be associated with abnormal activity of the complement cascade. Binding of recognition subunit (C1q) to a pathogen triggers self-activation of $\mathrm{C} 1 \mathrm{r}$, which in turn activates $\mathrm{C} 1 \mathrm{~s}$ that cleaves complement components $\mathrm{C} 2$ and $\mathrm{C} 4$, thereby initiating the complement cascade [12]. Kapferer-Seebacher et al. [7] reported that C1R pathogenic variants such as p.Cys309Trp, p.Cys338Arg, p.Cys358Phe, and p.Cys371Trp disrupt the paired cysteine residues involved in disulfide bonds that are important in stabilizing the catalytic and interaction domain of $\mathrm{C} 1 \mathrm{R}$, resulting in morphological changes in the rough endoplasmic reticulum. Thus, mutated $\mathrm{C} 1 \mathrm{R}$ is expected to alter the target specificity of the $\mathrm{C} 1$ complex. In the current case, a novel missense variant (p.Cys309Gly) was found, in the same residue of the previously reported variant (p.Cys309Trp) [7]. Although we did not perform in vivo or in vitro functional studies on the current patient, we expect p.Cys309Gly to be pathogenic based on the location of the residue, population data, and in silico analysis.

To our knowledge, multiple white matter lesions in the brain have only been reported in one other case of EDS VIII, which was not genetically confirmed [8]. On the other hand, brain inflammation due to abnormal activation of complement pathway has been observed in other more common demyelinating or neurodegenerative diseases such as Alzheimer disease and multiple sclerosis. In Alzheimer disease, the fibrillar amyloid plaques lead to complement activation, subsequently causing an acceleration of local inflammation and neuronal injuries [13]. In the type Il pattern of multiple sclerosis, complements and autoantibodies contribute to demyelination by complement activation in the blood vessels of white matter [14]. Thus, the brain abnormalities in EDS VIII might be associated with the alteration of complement and collagen processing. Further molecular and in vivo evidence is needed to validate this association between altered complement activity and brain lesions. Although there was no definite neurological symptom in the current patient, long-term observation is warranted for the development of neurological manifestation. As brain abnormalities have not been surveyed in EDS VIII, further evaluation of neurological manifestations with brain imaging studies would be necessary for more patients with EDS VIII.

\section{Acknowledgements}

This research was supported by the Bio \&t Medical Technology Development Program of the National Research Foundation (NRF) and funded by the Korean government (MSIP; MOHW)
(NRF-2016M3A9B4915706).

\section{References}

1. Malfait F, Francomano $C$, Byers $P$, Belmont J, Berglund B, Black J, et al. The 2017 international classification of the Ehlers-Danlos syndromes. Am J Med Genet C Semin Med Genet 2017;175:8-26.

2. Beighton $P$, De Paepe A, Steinmann B, Tsipouras P, Wenstrup RJ. Ehlers-Danlos syndromes: revised nosology, Villefranche, 1997. Ehlers-Danlos National Foundation (USA) and Ehlers-Danlos Support Group (UK). Am J Med Genet 1998;77:31-7.

3. Brady AF, Demirdas S, Fournel-Gigleux S, Ghali N, Giunta C, KapfererSeebacher I, et al. The Ehlers-Danlos syndromes, rare types. Am J Med Genet C Semin Med Genet 2017;175:70-115.

4. Stewart RE, Hollister DW, Rimoin DL A new variant of Ehlers-Danlos syndrome: an autosomal dominant disorder of fragile skin, abnormal scarring, and generalized periodontitis. Birth Defects Orig Artic Ser 1977;13:85-93.

5. Rahman N, Dunstan M, Teare MD, Hanks S, Douglas J, Coleman K, et al. Ehlers-Danlos syndrome with severe early-onset periodontal disease (EDS-VIII) is a distinct, heterogeneous disorder with one predisposition gene at chromosome 12p13. Am J Hum Genet 2003;73:198204.

6. Reinstein E, DeLozier CD, Simon Z, Bannykh S, Rimoin DL, Curry CJ. Ehlers-Danlos syndrome type VIII is clinically heterogeneous disorder associated primarily with periodontal disease, and variable connective tissue features. Eur J Hum Genet 2013;21:233-6.

7. Kapferer-Seebacher I, Pepin M, Werner R, Aitman TJ, Nordgren A,

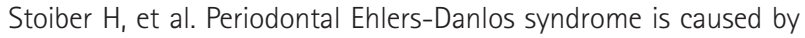
mutations in $\mathrm{C} 1 \mathrm{R}$ and $\mathrm{C} 1 \mathrm{~S}$, which encode subcomponents $\mathrm{C} 1 \mathrm{r}$ and C1s of complement. Am J Hum Genet 2016;99:1005-14.

8. Spranger S, Spranger M, Kirchhof $K_{1}$ Steinmann B. Ehlers-Danlos syndrome type VIII and leukodystrophy. Am J Med Genet 1996;66:23940.

9. Richards S, Aziz N, Bale S, Bick D, Das S, Gastier-Foster J, et al. Standards and guidelines for the interpretation of sequence variants: a joint consensus recommendation of the American College of Medical Genetics and Genomics and the Association for Molecular Pathology. Genet Med 2015;17:405-24.

10. Moore MM, Votava JM, Orlow SJ, Schaffer JV. Ehlers-Danlos syndrome type VIII: periodontitis, easy bruising, marfanoid habitus, and distinctive facies. J Am Acad Dermatol 2006;55:S41-5.

11. George SM, Vandersteen A, Nigar E, Ferguson DJ, Topham EJ, Pope FM. Two patients with Ehlers-Danlos syndrome type VIII with unexpected hoarseness. Clin Exp Dermatol 2016;41:771-4.

12. Cooper NR. The classical complement pathway: activation and 
regulation of the first complement component. Adv Immunol 1985;37:151-216

13. Shen $Y$, Lue $L$, Yang L, Roher A, Kuo Y, Strohmeyer R, et al. Complement activation by neurofibrillary tangles in Alzheimer's disease. Neurosci Lett 2001;305:165-8.
14. Brink BP, Veerhuis $R$, Breij EC, van der Valk P, Dijkstra CD, Bö L. The pathology of multiple sclerosis is location-dependent: no significant complement activation is detected in purely cortical lesions. J Neuropathol Exp Neurol 2005;64:147-55. 